\title{
Decentralized control of sound radiation using iterative loop recovery
}

\author{
Noah H. Schiller ${ }^{\mathrm{a}}$ and Randolph H. Cabell \\ NASA Langley Research Center, Structural Acoustics Branch, \\ Mail Stop 463, Hampton, VA 23681, USA \\ Chris R. Fuller \\ Virginia Tech, Department of Mechanical Engineering, \\ 131 Durham Hall, Blacksburg, VA 24061, USA
}

Short Title: Decentralized control using iterative loop recovery

(Dated: 08 September 2009)

\begin{abstract}
A decentralized model-based control strategy is designed to reduce low-frequency sound radiation from periodically stiffened panels. While decentralized control systems tend to be scalable, performance can be limited due to modeling error introduced by the unmodeled interaction between neighboring control units. Since bounds on modeling error are not known in advance, it is difficult to ensure the decentralized control system will be robust without making the controller overly conservative. Therefore an iterative approach is suggested, which utilizes frequency-shaped loop recovery. The approach accounts for modeling error introduced by neighboring control loops, requires no communication between subsystems, and is relatively simple. The control strategy is evaluated numerically using a model of a stiffened aluminum panel that is representative of the sidewall of an aircraft. Simulations demonstrate that the iterative approach can achieve significant reductions in radiated sound power from the stiffened panel without destabilizing neighboring control units.
\end{abstract}

PACS numbers: 43.50.Ki, 43.40.Vn

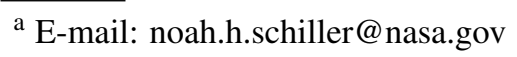




\section{INTRODUCTION}

Active sound and vibration control is desirable in many aerospace applications because of the potential size and weight savings relative to passive treatments such as poro-elastic foam. However despite the possible benefits, active noise control has been largely restricted to niche applications, focused on local regions in space or tonal disturbances. Global attenuation of broadband noise, which is desirable for large commercial aircraft, has only been demonstrated on laboratory scale systems. ${ }^{1-3}$ Significant implementation issues must be addressed to extend existing control strategies to larger systems such as full-scale commercial transports.

A promising approach uses structural control inputs to reduce the vibration and sound radiation from the fuselage. These structural inputs can be generated with compact piezoceramic actuators integrated in the structure, while feedback signals from accelerometers or piezoelectric transducers provide performance measures for the controller. While promising, this approach requires a large multiple-channel control system to reduce sound radiation from the entire fuselage. Although it is possible to use fully-coupled controllers, the approach is not practical on large systems with many inputs and outputs. Fully-coupled, or centralized, control requires a high level of connectivity, which can result in excessive wiring and weight. In addition, centralized control is computationally expensive, and can be particularly sensitive to transducer failures.

Since the fuselage of an aircraft is naturally segmented into bays by ring frames and stringers, one option is to assume that actuator/sensor pairs on the same bay are coupled while neglecting the coupling between transducers on neighboring bays. This approach, referred to as decentralized control, tends to be simple, computationally efficient, and scalable since each subsystem is controlled independently. Unfortunately if the neglected coupling between neighboring bays is significant, the approach can compromise both the stability and performance of the control system. ${ }^{4}$ As a result, there has been considerable interest in inherently robust control strategies, such as direct velocity feedback. ${ }^{5,6}$ These strategies allow for independent design and implementation of each control unit without concern for the global stability of the system. For example, it can be shown 
that if an actuator and sensor are collocated and dual, then any passive control law will guarantee the unconditional stability of the closed-loop system. ${ }^{7}$ Unfortunately, real transducer pairs are never perfectly collocated and dual, which can create high-frequency stability problems. In addition, the conservative nature of this control strategy tends to sacrifice performance in exchange for robust stability, hence the approach is referred to as low-authority control.

High-authority control, on the other hand, tends to sacrifice robust stability in exchange for closed loop performance. One such approach, active structural acoustic control (ASAC), uses structural control inputs to reduce the overall radiated sound power. ${ }^{8,9}$ The advantage of ASAC is that it can require fewer control channels and less control power than active vibration control strategies like direct velocity feedback. This is true because ASAC suppresses or restructures the structural modes that radiate most efficiently without expending energy on inefficient radiators. ${ }^{10}$ While this technique was originally developed for feedforward control applications, it can also be implemented using modern feedback control techniques. ${ }^{11}$ In particular, the structural acoustic control problem can be solved using linear quadratic Gaussian (LQG) theory. The advantage of LQG theory is that it provides analytical design procedures that can be used to calculate optimal control laws. While optimal control theory can be very powerful, the performance of the controller necessarily depends on the fidelity of the plant model from which the controller is designed. ${ }^{12}$ Poorly modeled dynamics can destabilize the closed-loop control system. As a result, optimal control has been used with limited success in decentralized configurations. ${ }^{4}$

The goal of this work is to combine the power of optimal control theory with the simplicity and scalability of decentralized control. In particular, this paper describes a scalable decentralized control approach based on an iterative improvement procedure. The paper begins with a brief description of decentralized control along with a discussion of its limitations. An optimal LQG control strategy is then described. Since LQG designs can have arbitrarily poor stability margins, two methods known as frequency shaping and loop transfer recovery (LTR) can be used to improve the robust stability and performance of the system. However, both techniques assume uncertainty 
bounds are known a priori, which is not always true. Therefore an iterative scheme is presented to account for the lack of a priori information. In addition to describing the control methodology, this paper also presents results from a numerical study used to evaluate the proposed approach.

\section{CONTROL METHODOLOGY}

The control methodology is discussed in this section. The overall objective is to reduce the sound power radiated from a stiffened panel using a scalable control strategy, hence only decentralized strategies are considered. A decentralized control strategy implies that each control unit is designed and implemented independently using information local to each bay. A notional view of the approach is shown in Fig. 1, where the control unit on each bay of the stiffened panel consists of a piezoceramic actuator and four accelerometers providing feedback signals. The following subsection discusses the consequences of using local control units on a coupled structure. Background information pertaining to LQG control and loop transfer recovery is then presented along with a description of the proposed control strategy.

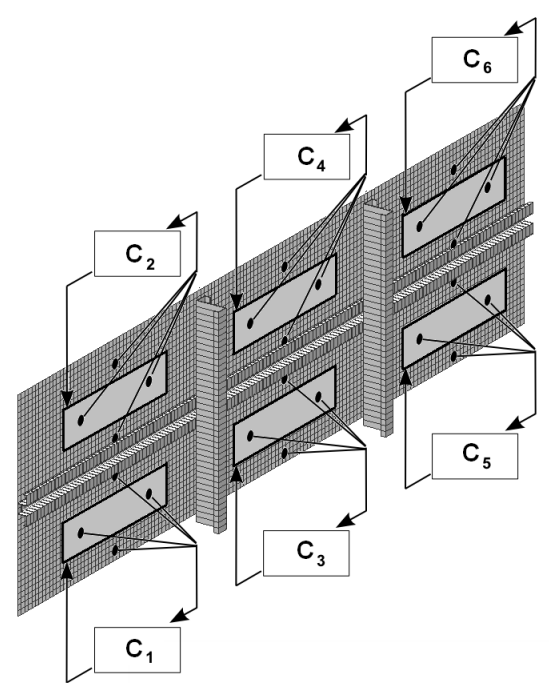

Figure 1: Decentralized control on a stiffened panel. 


\section{A. Decentralized Control}

A block diagram of a two-channel decentralized control system, representing two of the six control units depicted in Fig. 1, is shown in Fig. 2(a). The control input to bay 1, $u_{1}$, is based on sensor response $y_{1}$ only, while input $u_{2}$ on bay 2 is based on sensor response $y_{2}$ only. The grey box in the figure represents the actual structure, or plant, which can be written in matrix form as

$$
G=\left[\begin{array}{ll}
G_{11} & G_{12} \\
G_{21} & G_{22}
\end{array}\right]
$$

where $G_{i j}$ denotes the transfer function from the $i^{t h}$ actuator to the $j^{t h}$ sensor. In the context of this work, the diagonal terms of $G$ model the response between actuator/sensor pairs on the same bay, while the off-diagonal terms capture the coupling between actuators and sensors on different bays. Decentralized control is particularly effective if the plant is diagonally dominant (i.e. $\left.G_{11} G_{22}>>G_{12} G_{21}\right)$ since the cross-coupling between the $i^{t h}$ input and the $j^{\text {th }}$ output is neglected during the design process. Cross-coupling can have the effect of increasing the uncertainty in the dynamics of the local control loop on each bay. As an illustration, the block diagram in Fig. 2(a) can be rearranged as shown in Fig. 2(b), where the controller on bay 1 is represented as additional dynamics between the control input and sensor response on bay 2. Notice that while the controller $C_{2}$ is designed for the nominal subsystem model $G_{22}$, the cross-coupling terms $\left(G_{12}\right.$ and $\left.G_{21}\right)$ and the controller $C_{1}$ introduce an additional path from $u_{2}$ to $y_{2}$. Therefore, the combined model from $u_{2}$ to $y_{2}$ is

$$
G_{2}=G_{22}+\Delta_{A 1}
$$

where $\Delta_{A 1}=G_{21} C_{1} G_{12} /\left(1-C_{1} G_{11}\right)$ is the additive error introduced by $C_{1}$. If the local control system designed for $G_{22}$ is not robust to the modeling error introduced by $C_{1}$, then the coupled system will be unstable. Therefore effective decentralized control requires local controllers that are robust to uncertainty created by neighboring controllers.

Although Eq. 2 expresses the influence of a neighboring controller in terms of additive uncer- 


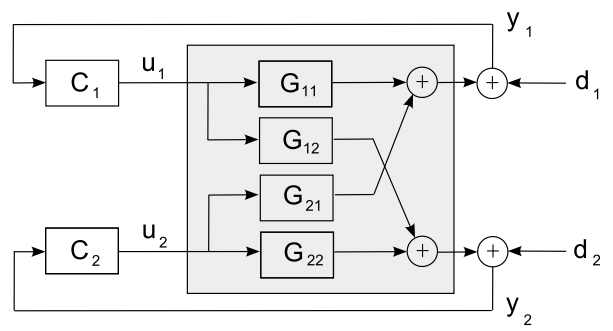

(a)

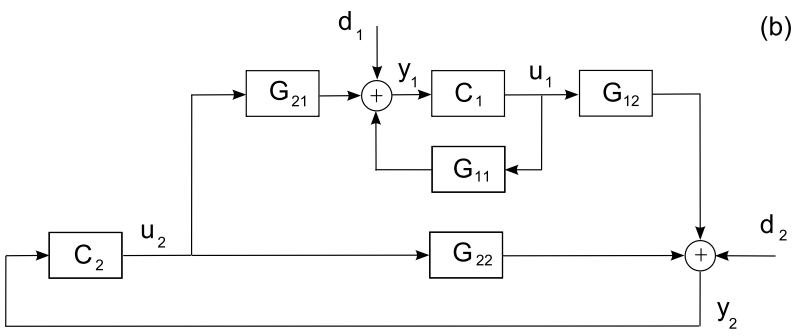

Figure 2: Standard block diagram of a two-channel decentralized control system (top), and an alternative representation highlighting the additional path from $u_{2}$ to $y_{2}$ through $C_{1}$ (bottom).

tainty, a more useful representation is in terms of multiplicative uncertainty, which is obtained by rewriting Eq. 2 as

$$
G_{2}=G_{22}\left(1+\Delta_{M 1}\right)
$$

where $\left(1+\Delta_{M 1}\right)$ is the multiplicative error, and the multiplicative uncertainty $\Delta_{M 1}$ is defined as $\Delta_{M 1}=\Delta_{A 1} / G_{22}$. In this example,

$$
\Delta_{M 1}=\left(\frac{G_{21} G_{12}}{G_{11} G_{22}}\right)\left(\frac{C_{1} G_{11}}{1-C_{1} G_{11}}\right) .
$$

Notice that the first term in parentheses provides a measure of the diagonal dominance of the plant. This term will be large if the product of the cross-coupling terms is large with respect to the product of the diagonal terms. The second term in Eq. 4 is the complementary sensitivity function, $T_{1}$. Robust stability is obtained when $\Delta_{M 1}$ is small. Hence, the control system designed for subsystem 2 must be robust at frequencies where the plant is not diagonally dominant and at frequencies where $T_{1}$ is large. Unfortunately since decentralized control units are designed using only local information, there is no way of knowing the cross-coupling terms or the complementary sensitivity function at design time. 
In addition to introducing dynamics that are not included in the local plant model, neighboring control loops also change the characteristics of the disturbance. Figure 2(b) shows that the sensor response $y_{2}$ contains contributions from disturbance $d_{2}$ and $d_{1}$, due to the controller $C_{1}$ on bay 1 . As a result, the disturbance at $y_{2}$ becomes $d_{2}+d_{1} C_{1} G_{12} /\left(1-C_{1} G_{11}\right)$. Disturbance variations are undesirable because the controller is typically designed to be optimal with respect to a specific disturbance. If the disturbance changes due to a controller on a neighboring bay, then the closedloop system may not be optimal with respect to the actual disturbance, thereby degrading closedloop performance. In summary, neighboring control loops introduce both modeling error and disturbance variations.

While it is important to fully appreciate the problems introduced by decentralized hierarchies, the next step is to incorporate this understanding into the design process. How do we design decentralized control units that meet our performance requirements and tolerate the modeling error and disturbance variations introduced by neighboring control units? The solution presented in this paper relies on LQG control theory, which is summarized in the following section.

\section{B. Linear quadratic Gaussian (LQG) control}

LQG controllers are designed by independently solving optimal state regulation and state estimation problems. ${ }^{13}$ The state regulation problem is solved by finding the optimal feedback gain matrix, $K$, that minimizes a quadratic function containing performance and control effort terms. Performance is expressed in terms of the state vector, $x$, while the control effort is expressed in terms of the input vector, $u$. Unfortunately the full state vector is rarely known in practice, and hence is usually reconstructed using a stochastic estimator, as shown in Fig. 3. In this diagram the plant is represented as

$$
\begin{aligned}
\dot{x} & =A x+B u+E w \\
y_{v} & =C x+v
\end{aligned}
$$


where $x$ is the state vector, $u$ is the input, $A$ is the state matrix, $B$ is the input matrix, $C$ is the output matrix, $E$ is the noise input matrix, $y_{v}$ is the plant output, $w$ is the disturbance, and $v$ is measurement noise. The estimator consists of a model of the plant with an additional feedback loop used to ensure the state estimates, $\hat{x}$, converge to $x$ sufficiently fast. The feedback signal is generated by multiplying the measurement error $y_{v}-\hat{y}$ by the observer gain matrix $L$. Since the feedback signal is generated using the noisy output measurement $y_{v}$, increasing the magnitude of $L$ amplifies measurement noise. Therefore, the optimal choice of $L$ involves a trade-off between measurement noise sensitivity and convergence speed.

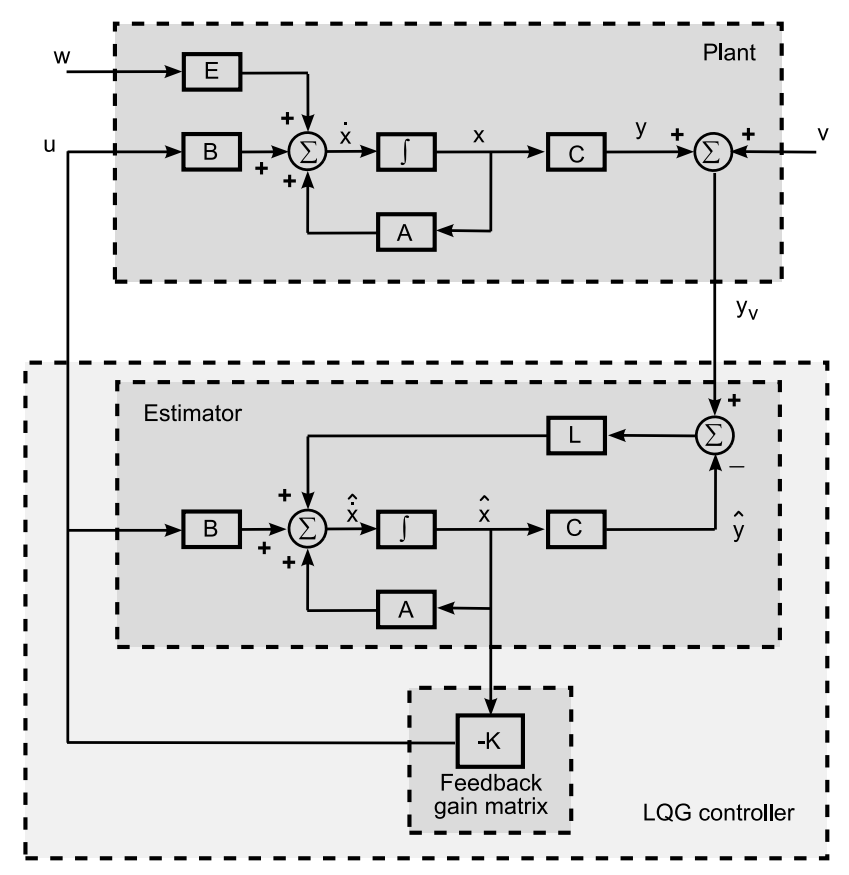

Figure 3: LQG diagram.

\section{Loop transfer recovery}

While full-state feedback has guaranteed stability margins, incorporation of the estimator into the loop can result in arbitrarily poor stability margins due to errors in the state estimates. ${ }^{12}$ In response to this problem, loop transfer recovery (LTR) was developed by Doyle and Stein ${ }^{14}$ to asymptotically "recover" the robustness properties of the full-state feedback design. As the name 
implies, the robustness of the regulator is recovered by making the LQG loop transfer function ${ }^{15}$

$$
H_{L Q G}=-K(s I-A+B K+L C)^{-1} L C(s I-A)^{-1} B
$$

approach that of the state regulator

$$
H_{L Q R}=-K(s I-A)^{-1} B
$$

This is achieved by modifying the design of the state estimator by injecting fictitious noise at the plant input, as depicted by $z$ in Fig. 4. If the plant is stabilizable, completely observable, time-invariant and also minimum phase, then the LQG design will asymptotically recover the characteristics of the state regulator as the amplitude of $z$ approaches infinity. ${ }^{13}$

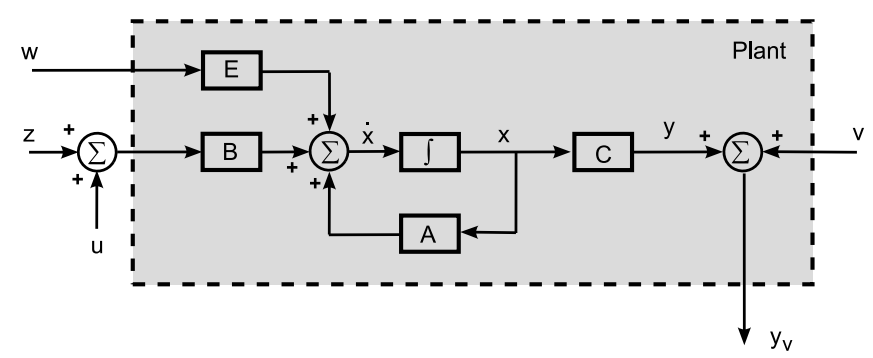

Figure 4: Plant model with fictitious noise, $z$, injected at the plant input.

However there are practical limitations associated with the loop recovery approach. For instance, full loop recovery relies on pole-zero cancellations, and hence is only guaranteed if the original plant is minimum phase. Because non-collocated transducers are rarely minimum phase, full recovery is rarely possible. Fortunately partial loop recovery, which is achieved by incrementally increasing the amplitude of the fictitious input noise, is often adequate to obtain an acceptable design that accounts for plant uncertainty.

Partial loop recovery can also be obtained in specific frequency bands by adding fictitious noise in frequency bands where improved stability margins are required. ${ }^{16}$ This enables different performance/robustness trade-offs in different frequency bands. ${ }^{17}$ Frequency shaped loop recovery can also be obtained by adding noise through the disturbance path. This is possible because the estimator design only depends on the ratio of process to measurement noise, ${ }^{13}$ so fictitious noise 
at either the plant input or disturbance increases the ratio of process to measurement noise. In both cases, the magnitude of the estimator gain matrix L will increase, resulting in partial loop recovery.

\section{Iterative loop recovery}

The control strategy described in this paper relies on frequency shaped loop recovery to account for changes to the dynamics and disturbance caused by control systems on neighboring bays. The control system on each bay is assumed to be non-minimum phase, making full loop recovery impossible. Therefore frequency shaped loop recovery is implemented by increasing the amplitude of the disturbance model in select frequency bands where the interaction between neighboring control units destabilizes the control system. Since it is difficult to predict the frequency bands where destabilizing interactions will occur, ${ }^{18}$ an iterative approach involving disturbance estimation and redesign of the LQG controller is used.

Figure 5 depicts the proposed control strategy which is implemented independently on each bay. The dynamics of the $i^{t h}$ bay, are denoted $G_{i}(z)$. This bay is excited by a disturbance $d_{i}(n)$, which on an aircraft could correspond to a combination of turbulent boundary layer excitation, propulsion noise, and aerodynamic forces due to irregular flow. A model of the physical system $\hat{G}_{i}(z)$ is assumed to be generated using a separate system identification process. An initial estimate of the disturbance is generated using the observed plant response, and then a nominal LQG controller is designed. Although the structure in the figure resembles internal model control, ${ }^{19}$ the input to the online controller $C_{i}(z)$ is the observed error signal $y_{i}(n)$ instead of the disturbance estimate $\hat{d}_{i}(n)$.

The disturbance estimate can be expressed as

$$
\hat{d}_{i}(z)=u_{i}(z)\left(G_{i}(z)-\hat{G}_{i}(z)\right)+d_{i}(z) .
$$

If the plant model is perfect, then the estimate will accurately track changes in the disturbance (i.e. $\left.\hat{d}_{i}(n)=d_{i}(n)\right)$. However it is important to consider the case when $\hat{G}_{i}(z) \neq G_{i}(z)$. In frequency bands where modeling error is destabilizing the amplitude of the disturbance estimate will 
be larger than the initial disturbance estimate (i.e. $\left|\hat{D}_{i}(k)\right|>\left|\hat{D}_{i}^{\prime}(k)\right|$, where $\hat{D}_{i}(k)$ is the current disturbance estimate and $\hat{D}_{i}^{\prime}(k)$ is the initial disturbance estimate). Although increasing the amplitude of the disturbance model tends to improve robustness by exploiting loop recovery, decreasing the amplitude can have the opposite effect and make the LQG controller more sensitive to modeling error. Therefore the disturbance model is updated by taking the maximum of both the current disturbance estimate and the previous disturbance model on a frequency-by-frequency basis.

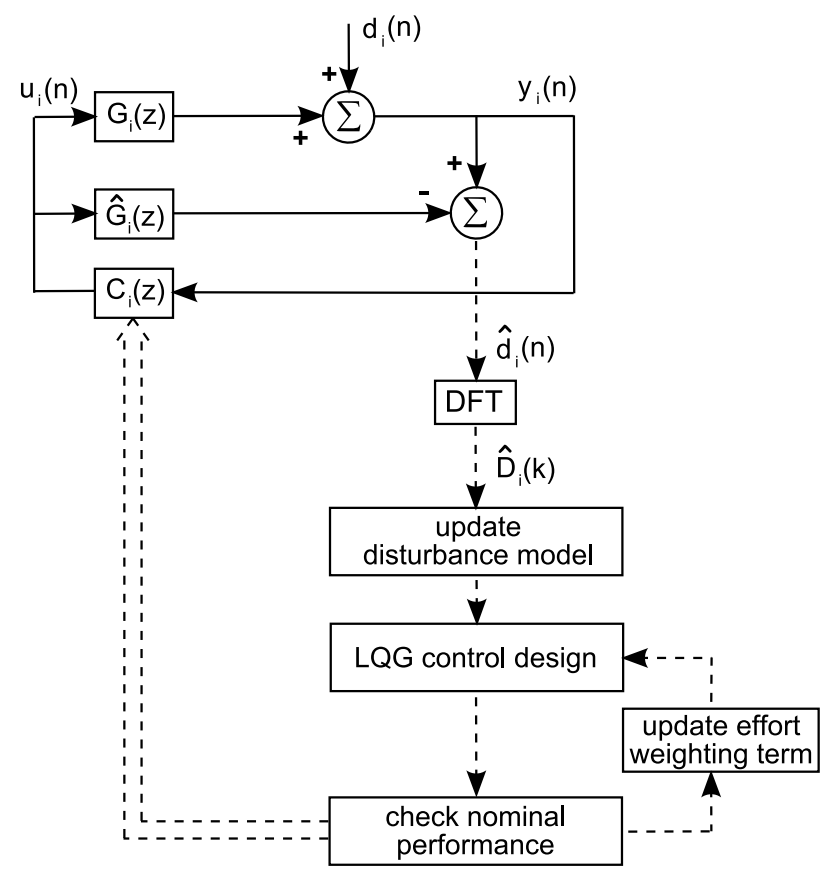

Figure 5: A diagram of the iterative control strategy.

In order to implement this procedure, the spectral density of the disturbance is estimated as

$$
S_{d d}(k)=\hat{D}_{i}(k) \hat{D}_{i}^{*}(k)
$$

where $\hat{D}_{i}(k)$ is the discrete Fourier transform of one record of the disturbance estimate, $\hat{D}_{i}^{*}(k)$ is the complex conjugate of $\hat{D}_{i}(k)$, and $k$ is the discrete frequency index. The spectral density of the new disturbance model $S_{p p_{\text {new }}}$ is then computed as

$$
S_{p p_{\text {new }}}(k)=(1-\alpha) \max \left[S_{d d}(k), S_{p p_{\text {old }}}(k)\right]+\alpha S_{d d}(k) \quad \text { for all } \quad k
$$

where $S_{p p_{\text {old }}}(k)$ is the spectral density of the old disturbance model, and $\alpha$ is a leakage parameter. 
Note that if the leakage parameter is set to zero, then the disturbance model can only increase. However, a leakage parameter of 0.1 is used in this work to track slow changes in the actual disturbance. The leakage parameter reduces the magnitude of the disturbance model if the disturbance estimate is consistently low.

Note that $S_{p p_{\text {new }}}$ is the spectral density of the desired disturbance model, not the model itself. Since the phase of the disturbance model is arbitrary for simple LQG systems, spectral factorization is used to fit the magnitude response with a stable and minimum phase plant. ${ }^{20}$

The iterative frequency-shaped LQG/LTR strategy accounts for parameter variations introduced by neighboring control loops, requires no communication between control systems, and is relatively simple. The following sections describe a numerical study that was used to evaluate the proposed approach. Note that this approach has also been successfully used in preliminary laboratory experiments, which are described in a separate report by Schiller. ${ }^{21}$

\section{NUMERICAL MODEL}

The stiffened flat panel depicted in Fig. 6 is used to represent the sidewall of an aircraft fuselage. While many academic studies consider simply-supported or clamped plates, those models are not representative of the aircraft fuselage at low frequencies where the structural wavelengths are long and the motion of both the panel and stiffeners is important. ${ }^{22}$ In addition, those simple models neglect the structural coupling between bays, which can destabilize decentralized control systems. ${ }^{4}$ The numerical model used here consists of a $1.27 \mathrm{~mm}$ thick flat clamped aluminum panel partitioned into six bays by a horizontal stringer and two vertical ring frames. The inverted hat-section stringer shown in Fig. 6 is made from $1.02 \mathrm{~mm}$ thick aluminum while the frames are made of $1.27 \mathrm{~mm}$ aluminum.

A finite element model of the stiffened panel was created using two-dimensional CQUAD4 elements to represent the aluminum plate and one-dimensional CBEAM elements for the stiffeners. The size of the elements was selected such that the model had at least 6 elements per flexural 


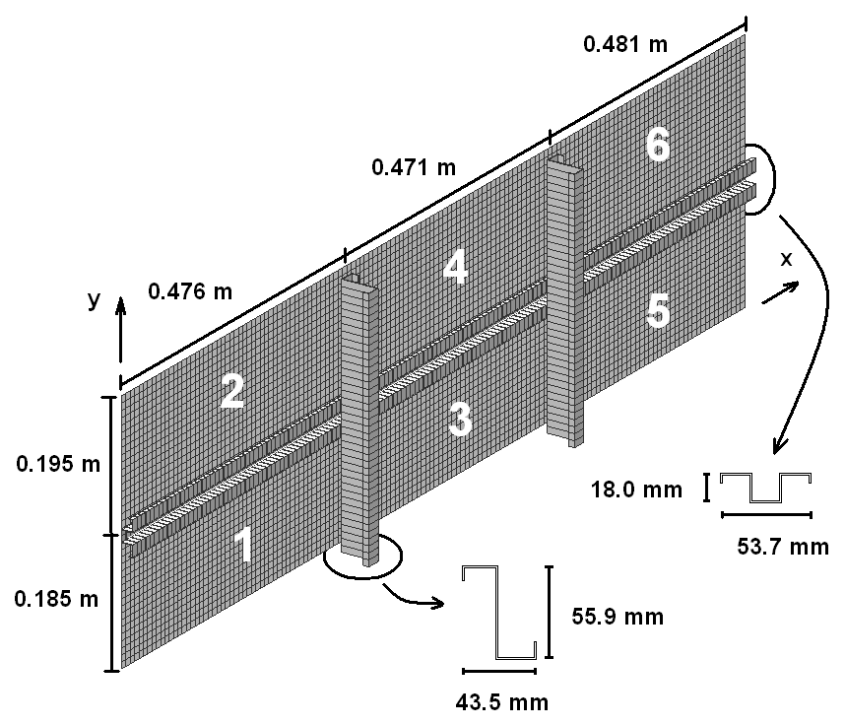

Figure 6: Diagram of the stiffened panel.

wavelength through $1.8 \mathrm{kHz}$. To achieve this, 143 elements were used in the x-direction and 38 elements were used in the y-direction. The elements along the edges of the panel were clamped, yielding a model with 26,484 degrees of freedom.

A normal modes analysis was used to identify the generalized mass and stiffness matrices, and to extract the 200 lowest frequency transverse modes. This was necessary to capture the dynamics through $1.8 \mathrm{kHz}$. The generalized mass and stiffness matrices were then used to create a statespace model of the structure. For this study, a modal damping ratio of $1 \%$ was used for all modes. The structural model was then augmented to include the dynamics of the piezoelectric actuators as described by Clark et al. ${ }^{23}$ The sound power radiated by the structure was estimated using a reduced order radiation model ${ }^{24}$ containing 6 radiation modes, which account for more than $99 \%$ of the power radiated from the structure below $1 \mathrm{kHz}$. The final structural acoustic model contains 418 states describing 200 structural modes and 6 radiation modes. The structure is excited by a broadband spatially correlated excitation, which is representative of a normally incident plane wave. This type of excitation was modeled using 150 correlated point loads distributed over the structure.

The control transducers consist of accelerometers and surface-mounted piezoceramic patches. 
Specifically, $0.07 \mathrm{~m}$ by $0.29 \mathrm{~m}$ piezoelectric actuators were mounted in the center of each bay, and four accelerometers were located in a diamond pattern around each actuator. When integrated, the summed response from each set of accelerometers provides an estimate of the volume velocity of the bay. This transducer configuration was selected based on the controller/transducer complexity work performed by Gibbs et al. ${ }^{3}$

\section{RESULTS AND DISCUSSION}

Numerical simulations are used to study the limitations of decentralized LQG control and evaluate the performance of iterative loop recovery. The results of two simulations are discussed. First, the need for iterative loop recovery is established by examining interactions between controllers on two neighboring bays. The advantage of disturbance estimation and loop recovery is demonstrated on these two bays. The performance of iterative loop recovery is then evaluated on a system with independent control units on each of the six bays of the panel. Since modern control systems are typically implemented digitally, all simulations are performed in discrete-time with a sample rate of $3 \mathrm{kHz}$.

In both simulations, the LQG controllers are designed by independently solving optimal state regulation and state estimation problems. The state regulator is found by calculating the optimal feedback gain matrix that minimizes a quadratic function containing performance and control effort terms. The performance variable is assumed to equal the sensed variable (i.e. the volume velocity estimate) while the magnitude of the control effort weighting term is used to vary the trade-off between performance and control effort. For these simulations, the measurement noise is assumed to have a flat magnitude of $-60 \mathrm{~dB}$ relative to $1 \mathrm{~m} / \mathrm{s}$, while disturbance models are used to capture the shape of the process noise. Since each controller has a single input and output, the phase of the disturbance model is arbitrary. Therefore disturbance models are initially generated by fitting the magnitude of the local open-loop response with 55th order minimum phase models. Although the full structural acoustic model contains 418 states, reduced order local control models 
are used to design each controller. Therefore, each LQG controller contains 130 states.

\section{A. Decentralized control of two bays}

Initial simulations are performed by implementing independent LQG controllers on two neighboring bays of the stiffened panel. The two bays and corresponding transducer layouts are depicted in Fig. 7. The power spectrum of the summed velocity on bay 3 due to the disturbance only (the open-loop response) is indicated by the black line in Fig. 8. When feedback control is implemented on bay 3 only, the corresponding closed loop response is indicated by the dash-dotted blue line in Fig. 8. The controller reduces the response, relative to the open loop response, near the resonances at 200,600, and $950 \mathrm{~Hz}$, and slightly increases the response around $1250 \mathrm{~Hz}$. The dashed red line in Fig. 8 shows the response on bay 3 when feedback control is simultaneously implemented on bays 3 and 4 . In this example, the interaction between the two control units causes excessive spillover at 175 and $1237 \mathrm{~Hz}$. In the context of this work, spillover is the undesired amplification of the response with respect to the open-loop response.

Adding a control unit on bay 4 introduces dynamics that are not included in the initial plant model of bay 3. The plant variations affect both the stability margins and performance of the control system. For instance, the left hand side of Fig. 9 shows the Nyquist diagram for the control unit on bay 3 from 0 to $177 \mathrm{~Hz}$. The dash-dotted blue line shows the polar plot of the open-loop frequency response, $G_{3}(s) C_{3}(s)$, where $G_{3}(s)$ is the nominal model of bay 3 . In this case, the control system has a gain margin of $4.5 \mathrm{~dB}$ and a phase margin of 35 degrees. The dashed red line shows the polar plot of $\widetilde{G}_{3}(s) C_{3}(s)$, where $\widetilde{G}_{3}(s)$ is the modified plant that includes the dynamics of the controller on bay 4 . The gain and phase margins of this system are only $-0.73 \mathrm{~dB}$ and 10 degrees, respectively. Although the coupled system is stable, excessive spillover is observed around $175 \mathrm{~Hz}$ (indicated in the figure with red circles) where the polar plot passes close to the Nyquist point $(-1,0)$. Similarly, the right hand side of Fig. 9 shows the Nyquist diagram from 1 to $1.25 \mathrm{kHz}$. Once again, the additional dynamics introduced by the controller on bay 4 destabilizes 

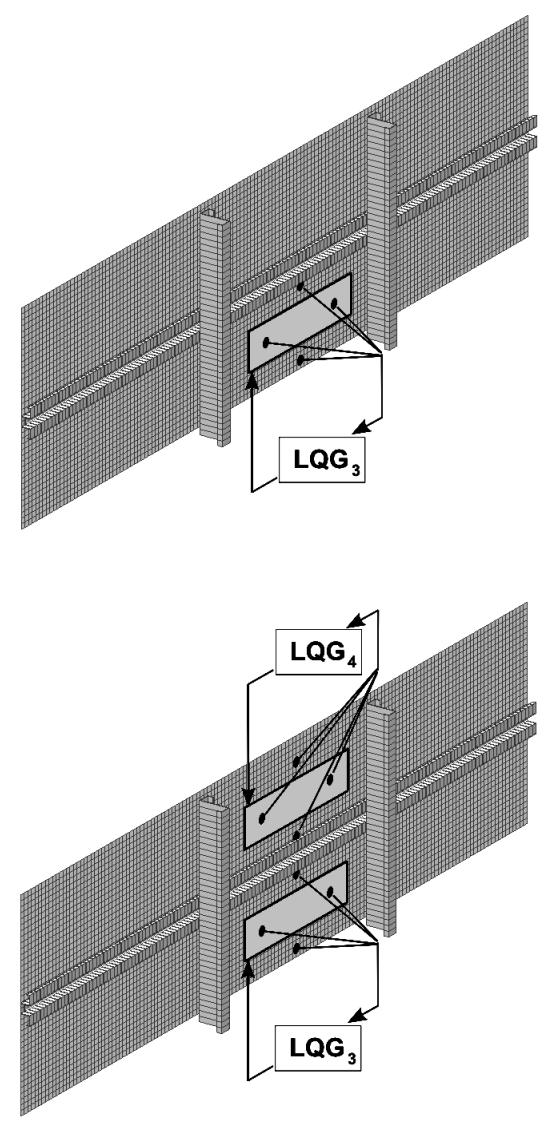

Figure 7: Transducer layout with control on bay 3 (top) and control on bays 3 and 4 (bottom).

the control system. The open-loop frequency response function of the combined system passes close to the Nyquist point at $1237 \mathrm{~Hz}$ (identified in the figure using red circles), which causes the large spike in the closed-loop response at that frequency.

In addition to introducing plant variations, the interaction between the two control units also changes the shape of the disturbance, as shown in Fig. 10. The thin black line shows the open-loop power spectrum of the summed velocity on bay 3 , which corresponds to the nominal disturbance spectrum for that bay. The dashed red line shows the power spectrum of the summed velocity on bay 3 with control only on bay 4, which shows the change in the disturbance due to the addition of a neighboring control unit. While changes in the disturbance spectrum do not affect the stability margins, they can affect closed-loop performance. Feedback controllers are typically designed such that the sensitivity function (output over the disturbance) is much less than unity over a small 


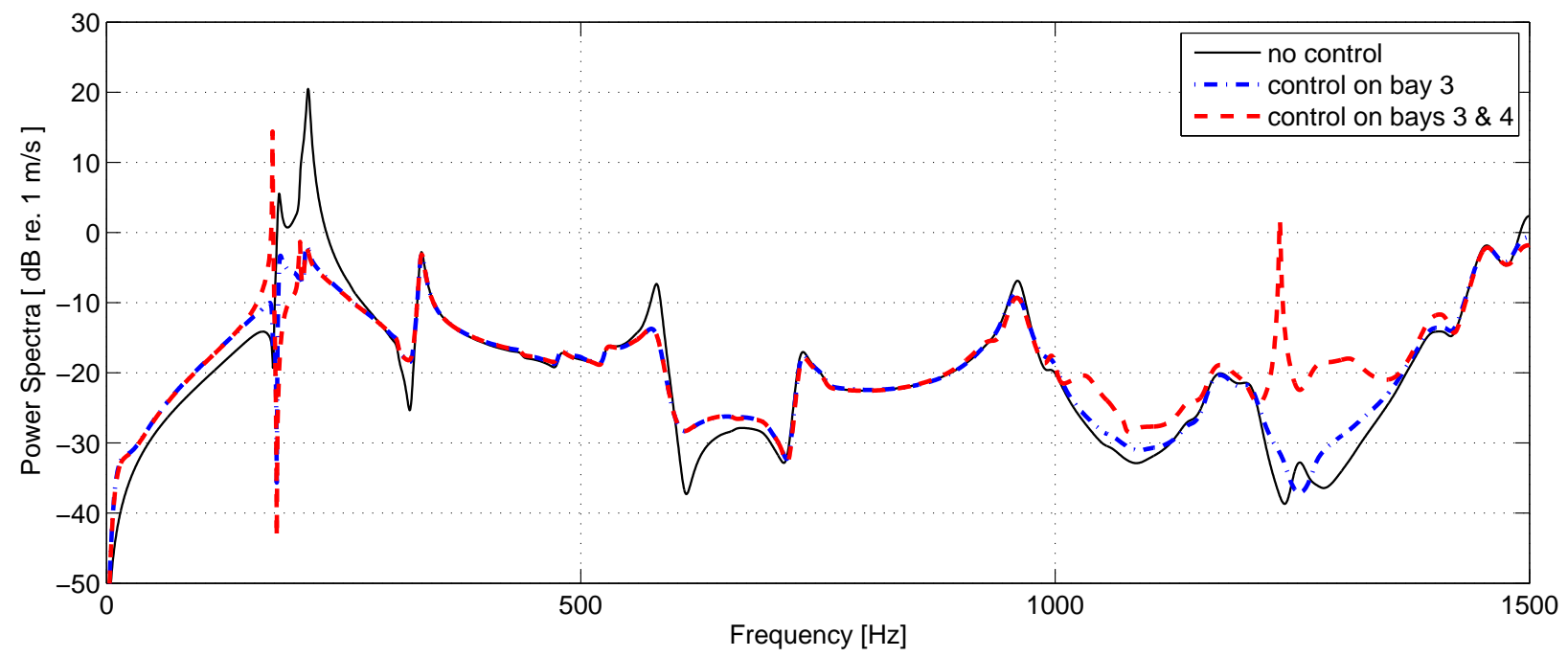

Figure 8: (Color online) Open and closed-loop response using decentralized LQG control.
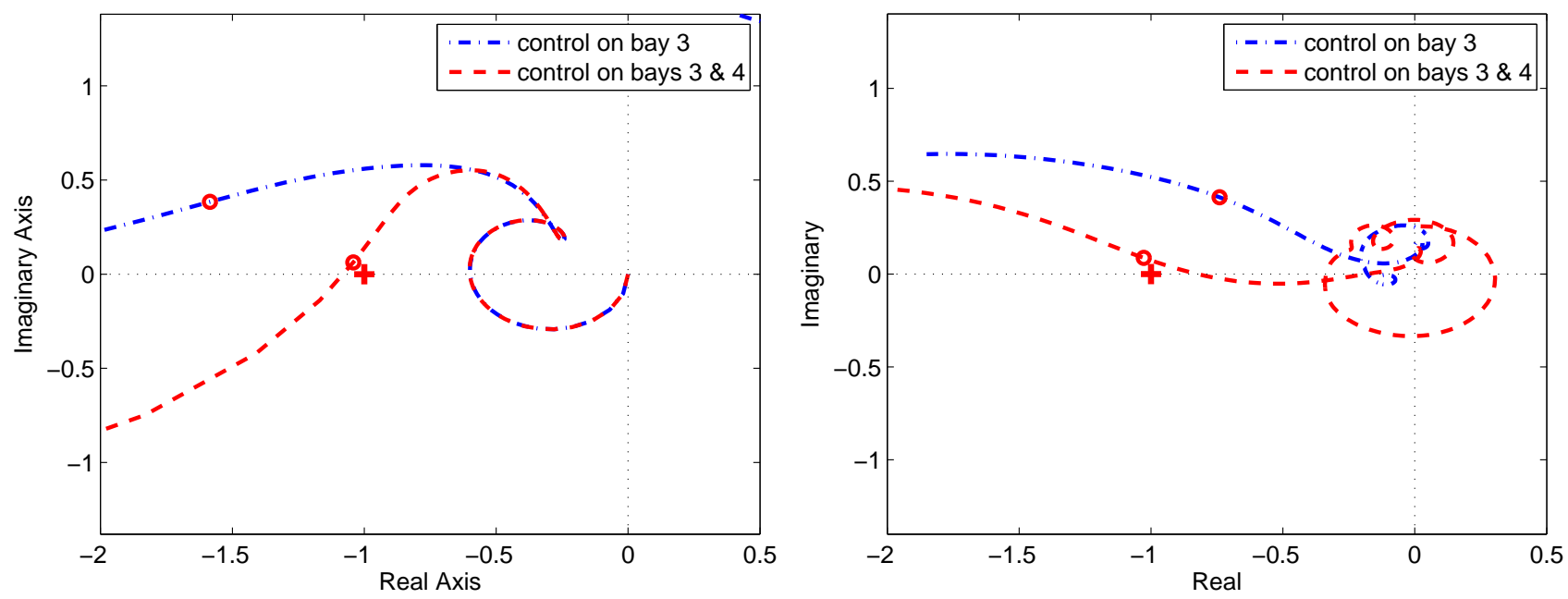

Figure 9: (Color online) Polar plots of the nominal open-loop frequency response function $G_{3}(s) C_{3}(s)$ (dash-dotted blue line) and the modified open-loop frequency response function $\widetilde{G}_{3}(s) C_{3}(s)$ (dashed red line) from 0 - $177 \mathrm{~Hz}$ (left) and from $1-1.25 \mathrm{kHz}$ (right).

bandwidth where the disturbance has significant energy in exchange for small increases over a large range of frequencies where the disturbance has little energy. Therefore changes in the shape of the disturbance model can degrade the closed-loop performance of the system.

To account for both plant and disturbance variations, the controller on bay 3 is updated using the online disturbance estimate. The online disturbance estimate, obtained with control on both bays 3 and 4, is shown with the dash-dotted cyan line in Fig. 10. Notice that the estimate closely 
tracks the actual disturbance (dashed red line) over much of the frequency band. However the disturbance estimate exceeds the actual disturbance at $175 \mathrm{~Hz}$ and $1237 \mathrm{~Hz}$ where the modeling error is destabilizing. Using Eq. 10, a new disturbance estimate is computed and then used to redesign the LQG controller. The resulting closed loop response, shown as the dash-dotted cyan line in Fig. 11, illustrates the benefit of using an updated disturbance model to account for plant and disturbance variations caused by neighboring controllers.

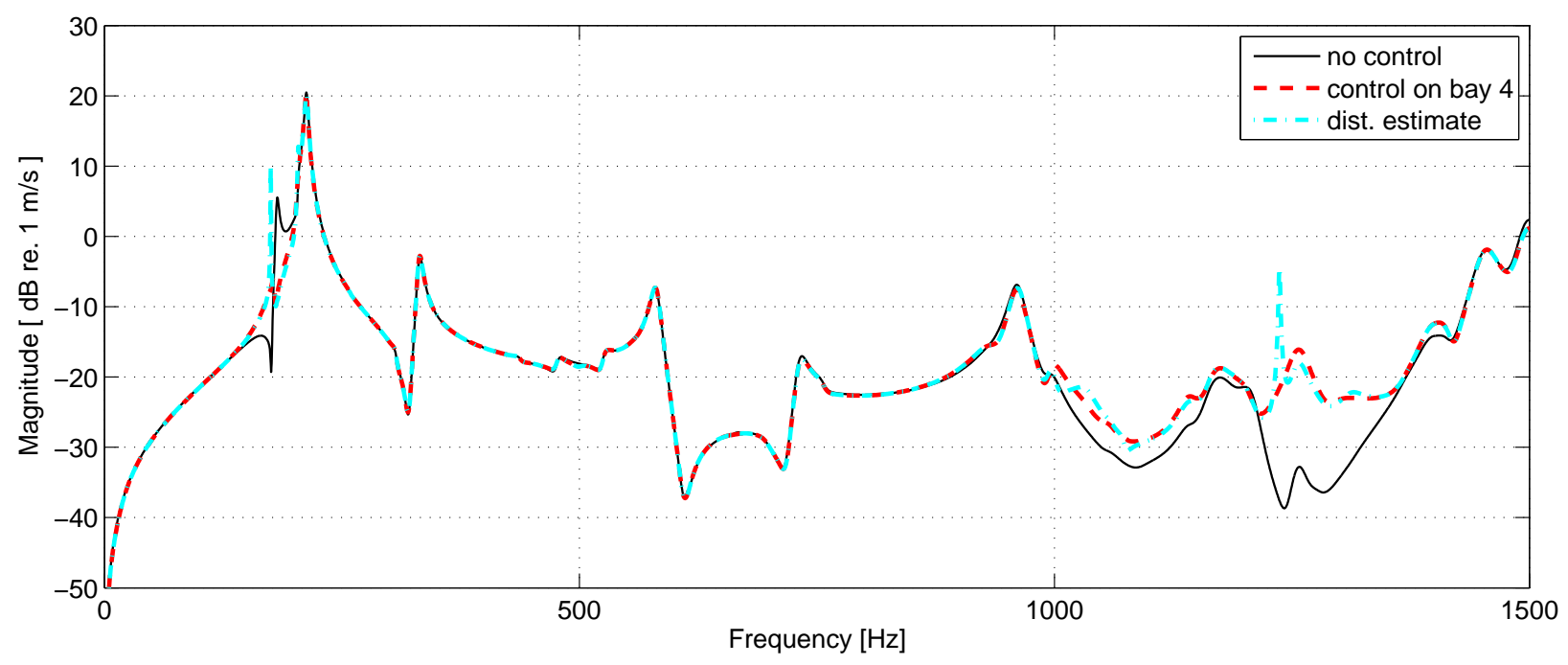

Figure 10: (Color online) Amplitude of the disturbance on bay 3.

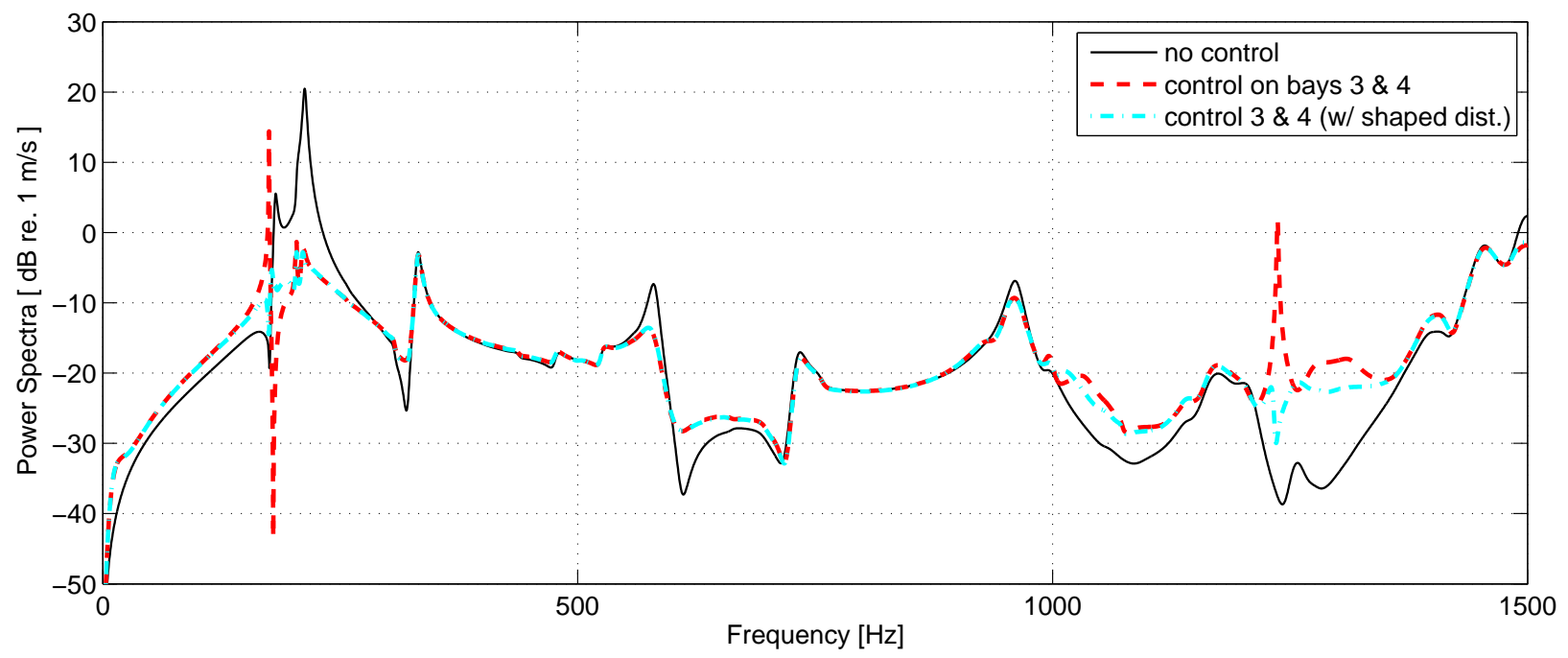

Figure 11: (Color online) Open and closed-loop response on bay 3 with controllers on two bays. 


\section{B. Decentralized control of six bays}

Simulations are also used to evaluate the full decentralized control system depicted in Fig. 12. In this case, all six controllers are designed independently without information pertaining to neighboring designs. Four different sets of controllers are evaluated beginning with relatively conservative LQG controllers and progressing to more aggressive designs (i.e. reduced effort weighting term). The open and closed-loop response (summed velocity) on bay 3 is shown in Fig. 13. Only the response of bay 3 is shown since similar trends are also observed on the other five bays. As the controllers become more aggressive, the closed-loop response deteriorates at 175, 205, and $1237 \mathrm{~Hz}$. The interaction between the six control units causes spillover at these frequencies, limiting achievable performance.

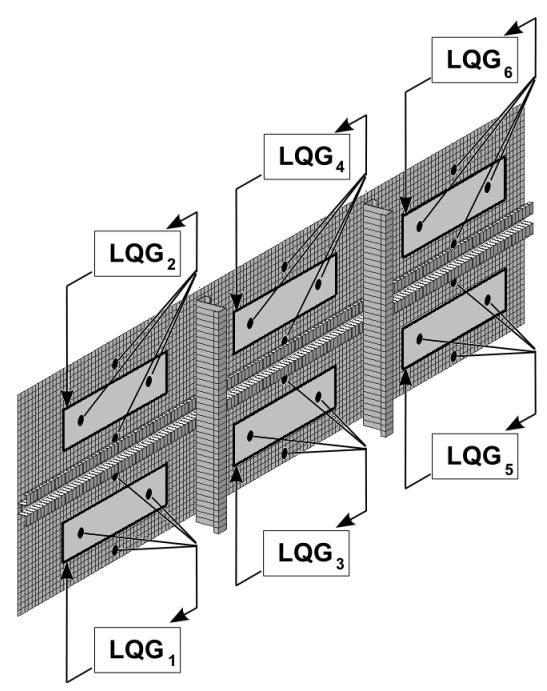

Figure 12: Decentralized control of 6 independent control units.

Figure 14 shows the open and closed-loop response on bay 3 when the iterative control approach is used. As in the previous example, relatively conservative LQG controllers are used initially. However instead of simply redesigning the controllers using a more aggressive effort weighting term, the disturbance models are also updated based on closed-loop system measurements. The redesigned controllers are then implemented and the update procedure is repeated. The 


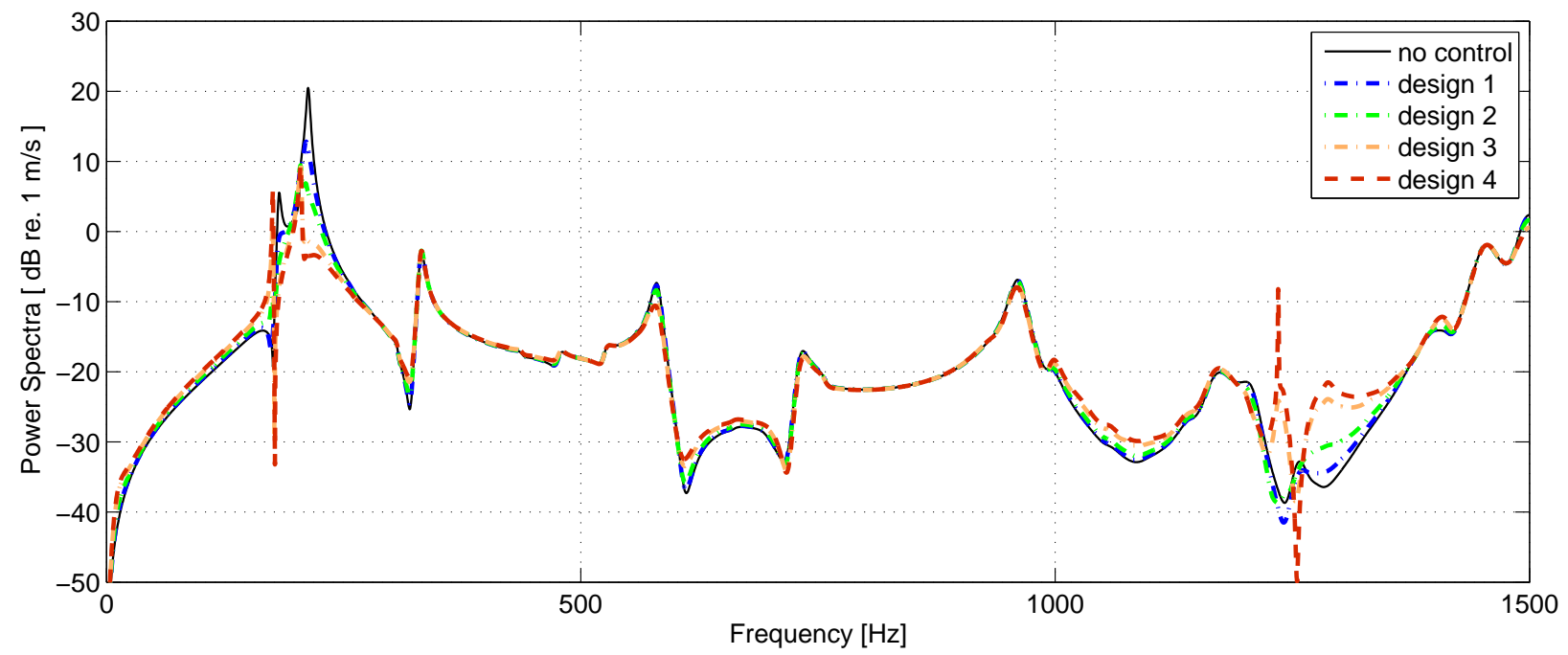

Figure 13: (Color online) Open and closed-loop response on bay 3 using decentralized LQG controllers on all six bays.

closed-loop responses corresponding to the first five design iterations are shown in Fig. 14. The final response, shown with the dashed brown line, achieves an integrated reduction of $13.7 \mathrm{~dB}$ from 50-1000 Hz without excessive spillover. While not shown, similar reductions are also achieved on the other five bays.

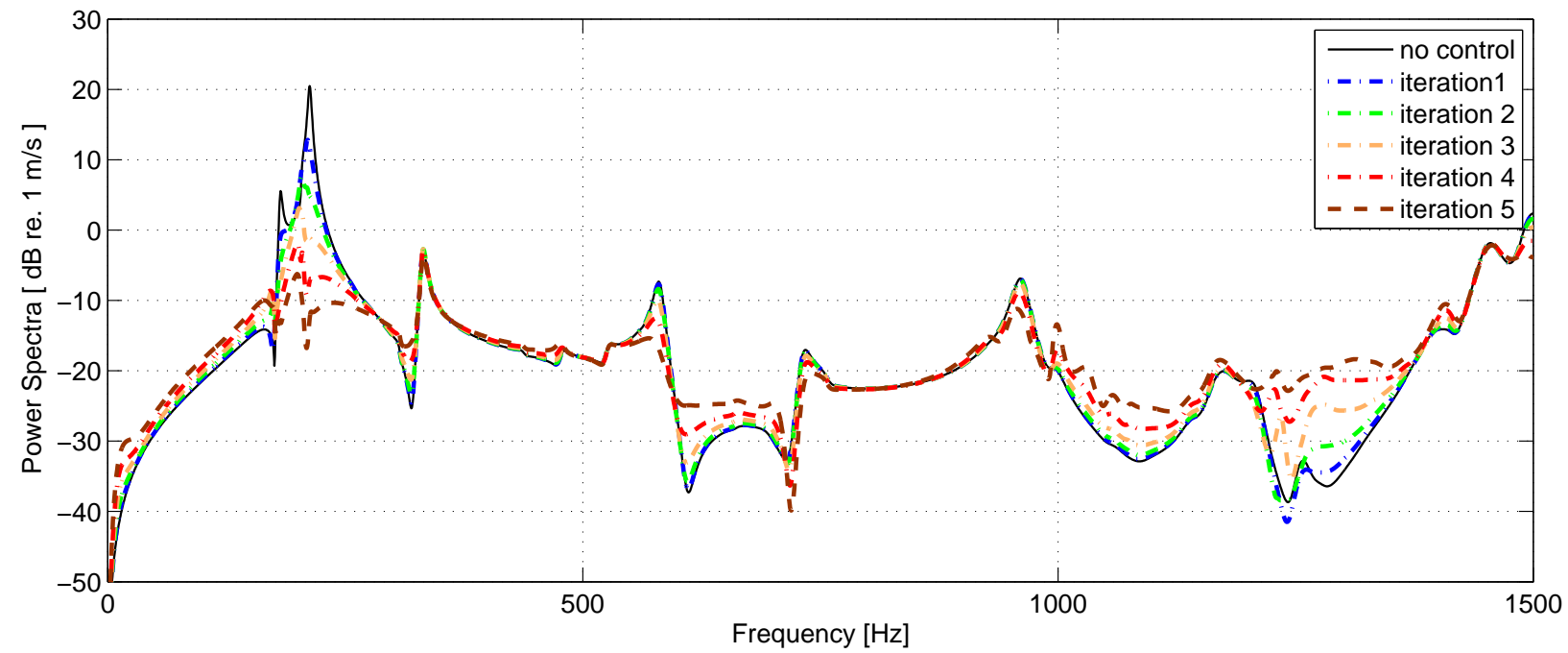

Figure 14: (Color online) Open and closed-loop response on bay 3 using iterative loop recovery on all six bays.

Finally consider the global performance of the control system in terms of the radiated sound 
power from the entire stiffened panel. Figure 15 compares the open-loop response, shown with the thin black curve, with the closed-loop responses achieved with the standard decentralized approach and the iterative strategy. The dash-dotted green curve corresponds to the standard decentralized approach in which the plant and disturbance models are designed based on open-loop measurements. Although this approach achieves a $5.7 \mathrm{~dB}$ integrated reduction from $50-1000 \mathrm{~Hz}$, performance is limited due to the destabilizing interaction between the local control units at 175 , 205, and $1237 \mathrm{~Hz}$. On the other hand iterative loop recovery, shown with the dashed brown line, achieves a $27 \mathrm{~dB}$ peak reduction and $7.7 \mathrm{~dB}$ integrated reduction in radiated sound power from $50-1000 \mathrm{~Hz}$ without large peaks caused by the interaction between local control units.

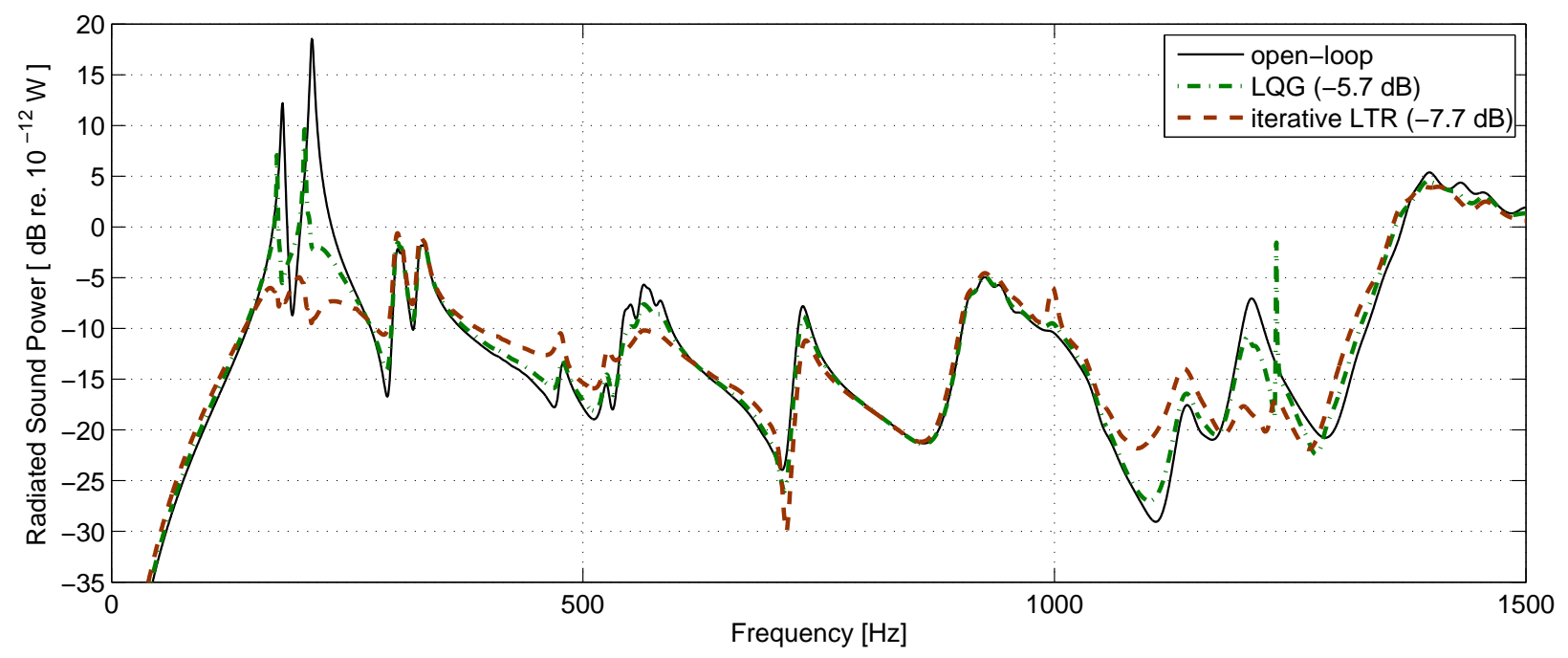

Figure 15: (Color online) Radiated sound power from the stiffened panel.

\section{CONCLUDING REMARKS}

Decentralized controllers introduce unavoidable errors due to the unmodeled coupling between subsystems. Since accurate uncertainty bounds are not known in advance, it is difficult to ensure the decentralized control system will be robust without making the controller overly conservative. Therefore an iterative approach is suggested, which utilizes frequency-shaped loop recovery. The approach accounts for modeling error introduced by neighboring control loops, requires no communication between subsystems, and is relatively simple. The approach is implemented by 
updating the disturbance model and redesigning the controller using local closed-loop measurements. This strategy was evaluated numerically using six independent control units mounted on a stiffened structure representative of the fuselage of an aircraft. Results demonstrate that updating the disturbance model based on closed-loop system measurements can improve the robust stability and performance of the control system with respect to standard decentralized strategies. In particular, the iterative control system achieved a $7.7 \mathrm{~dB}$ integrated reduction in radiated sound power from the stiffened aircraft-style panel.

\section{REFERENCES}

1 R. L. Clark and D. E. Cox, "Experimental demonstration of a band-limited actuator/sensor selection strategy for structural control,” J. Acoust. Soc. Am. 106, 3407-3414 (1999).

2 J. S. Vipperman and R. L. Clark, "Implications of using colocated strain-based transducers for output active structural acoustic control,” J. Acoust. Soc. Am. 106, 1392-1399 (1999).

${ }^{3}$ G. P. Gibbs, R. H. Cabell, and J. Juang, "Controller complexity for active control of turbulent boundary layer noise from panels," AIAA Journal 42, 1314-1320 (2004).

4 G. P. Gibbs and R. H. Cabell, "Simultaneous active control of turbulent boundary layer induced sound radiation from multiple aircraft panels," Proceedings of 8th AIAA/CEAS Aeroacoustics Conference, Breckenridge, $C O$ (2002).

5 S. J. Elliott, P. Gardonio, T. C. Sors, and M. J. Brennan, “Active vibroacoustic control with multiple local feedback loops,” J. Acoust. Soc. Am. 111, 908-915 (2002).

6 P. Gardonio and S. J. Elliott, "Smart panels with velocity feedback control systems using triangularly shaped strain actuators,” J. Acoust. Soc. Am. 117, 2046-2064 (2005).

7 M. J. Balas, "Direct velocity feedback control of large space structures," J. Guid. Control 2, 252-253 (1979).

8 C. R. Fuller, “Apparatus and method for global noise reduction,” U.S. Patent No. 4715599 (1987). 
9 C. R. Fuller and J. D. Jones, "Experiments on reduction of propeller induced interior noise by active control of cylinder vibration,” J. Sound Vib. 112, 389-395 (1987).

10 C. R. Fuller, S. J. Elliott, and P. A. Nelson, Active control of vibration (Academic Press, London, 1996).

11 W. T. Baumann, W. R. Saunders, and H. H. Robertshaw, "Active suppression of acoustic radiation from impulsively excited structures,” J. Acoust. Soc. Am. 90, 3202-3208 (1991).

12 J. C. Doyle, “Guaranteed margins for LQG regulators," IEEE Trans. Automat. Control AC-23, 756-757 (1978).

13 B. D. Anderson and J. B. Moore, Optimal Control: Linear Quadratic Methods (Prentice Hall, Englewood Cliffs, New Jersey, 1990).

14 J. C. Doyle and G. Stein, "Robustness with observers," IEEE Trans. Automat. Control AC-24, 607-611 (1979).

15 R. R. Bitmead, M. Gevers, and V. Wertz, Adaptive Optimal Control: The Thinking Man's GPC (Prentice Hall, Englewood Cliffs, New Jersey, 1990).

16 J. B. Moore, D. Gangsaas, and J. D. Blight, "Performance and robustness trades in lqg regulator design," Proceedings of the 20th IEEE Conference on Decision and Control, San Diego, California (1981).

17 G. Stein and M. Athans, "The LQG/LTR procedure for multivariable feedback control design,” IEEE Trans. Automat. Control AC-32, 105-114 (1987).

18 R. H. Cabell, D. E. Cox, and G. P. Gibbs, "Interaction metrics for feedback control of sound radiation from stiffened panels," Proceedings of the 44th AIAA/ASME/ASCE/AHS Structures, Structural Dynamics, and Matrials Conference, Norfolk, Virginia (2003).

19 M. Morari and E. Zafiriou, Robust process control (Prentice Hall, Upper Saddle River, New Jersey, 1989).

20 S. J. Elliott, Signal processing for active control (Academic Press, London, 2001).

21 N. H. Schiller, "Decentralized control of sound radiation from periodically stiffened panels," Ph.D. thesis, Virginia Tech, Blacksburg, Virginia (2007).

22 J. S. Mixson and J. F. Wilby, "Interior noise," Aeroacoustics of Flight Vehicles, Theory and Practice, 
edited by H. H. Hubbard (NASA Langley Research Center, Hampton VA, 1991), 271-355.

23 R. L. Clark, W. R. Saunders, and G. P. Gibbs, Adaptive Structures, Dynamics and Control (John Wiley and Sons, NY, 1998).

24 G. P. Gibbs, R. L. Clark, D. E. Cox, and J. S. Vipperman, "Radiation modal expansion: Application to active structural acoustic control," J. Acoust. Soc. Am. 107, 332-339 (2000). 


\section{Collected figure captions}

FIG. 1. Decentralized control on a stiffened panel.

FIG. 2. Standard block diagram of a two-channel decentralized control system (top), and an alternative representation highlighting the additional path from $u_{2}$ to $y_{2}$ through $C_{1}$ (bottom).

FIG. 3. LQG diagram.

FIG. 4. Plant model with fictitious noise, $z$, injected at the plant input.

FIG. 5. A diagram of the iterative control strategy.

FIG. 6. Diagram of the stiffened panel.

FIG. 7. Transducer layout with control on bay 3 (top) and control on bays 3 and 4 (bottom).

FIG. 8. (Color online) Open and closed-loop response using decentralized LQG control.

FIG. 9. (Color online) Polar plots of the nominal open-loop frequency response function $G_{3}(s) C_{3}(s)$ (dash-dotted blue line) and the modified open-loop frequency response function $\widetilde{G}_{3}(s) C_{3}(s)$ (dashed red line) from $0-177 \mathrm{~Hz}$ (left) and from $1-1.25 \mathrm{kHz}$ (right).

FIG. 10. (Color online) Amplitude of the disturbance on bay 3.

FIG. 11. (Color online) Open and closed-loop response on bay 3 with controllers on two bays.

FIG. 12. Decentralized control of 6 independent control units.

FIG. 13. (Color online) Open and closed-loop response on bay 3 using decentralized LQG controllers on all six bays.

FIG. 14. (Color online) Open and closed-loop response on bay 3 using iterative loop recovery on all six bays.

FIG. 15. (Color online) Radiated sound power from the stiffened panel. 